Multiple facilities are funded to assess the same parameters in several mouse strains under standardized conditions ${ }^{15}$. Unfortunately, most measurements occur only after the animals die.

Few studies track declines with ageing in living animals: bones thinning, hearts pumping less efficiently, or sluggishness in cognitive tasks. The first thorough paper on evaluating cardiovascular function in ageing mice was published barely five years ago ${ }^{16}$. Similarly, meetings on the basic biology of ageing concentrate on molecular mechanisms and pay scant attention to function.

Animals have evolved physiological redundancies, so manipulating a gene or signalling pathway does not necessarily change the function of an organ or organism. A drug that slows ageing should slow declines in several organ systems, but few laboratories can measure this in animals. One that does is the Healthspan Assessment Laboratory at the Mayo Clinic in Rochester, Minnesota. The lab quantifies muscle strength and cognitive impairment, and can measure body composition, metabolic rate, insulin sensitivity, motor coordination, bone density and exercise capacity. The Buck Institute for Research on Aging in Novato, California, (where S.M. works) has established similar resources. More facilities like this are needed.

\section{ADAPTING TOOLS}

Researchers need new methods to characterize function, particularly to track the same animals as they age, and before and after interventions. They need imaging tools and minimally invasive techniques, and those already established should be included in more experiments. Highest priority should be given to the assessment of motor and cognitive functions ${ }^{17}$ most likely to be recognized by drug regulators and industry. And, as in human studies, function should be characterized in several organs and tissues. Variability among animals, which increases with ageing, must also be accounted for.

Even studies that do assess function in animals often do so under conditions with little relevance to human ageing. Experiments on whether a drug improves cardiac function typically use young mice whose hearts have been purposely injured, rather than old mice with naturally aged hearts. To study the effects of obesity on the diseases of ageing, mice are fed a diet of up to $70 \%$ fat, with high proportions of trans and saturated fats; such diets bear little resemblance to typical human diets. Effects of inflammation are induced by molecules such as lipopolysaccharide at concentrations that would never be observed in normal physiology. Damage from oxidative stress, which accumulates over the years in humans, is rapidly induced with toxic chemicals such as paraquat.

Translational studies must be different.

\title{
MISSING LINKS
}

Several measures of how body function in humans changes with age can be adapted to rodents. Other tests not shown here include assessments of heart function (echocardiography), cognition (behavioural tests), aerobic-exercise capacity and metabolic rate.

\section{GRIP-STRENGTH TEST}

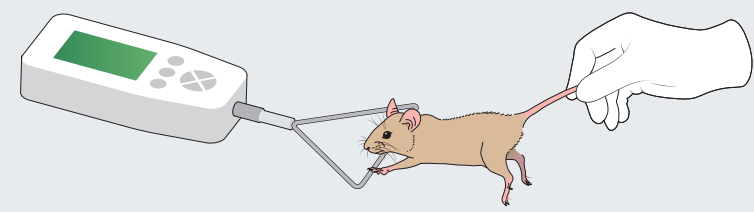

COORDINATION TEST

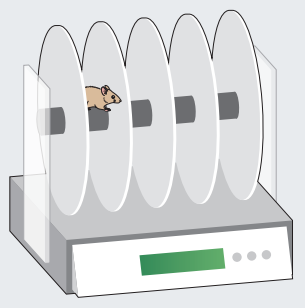

BODYSCAN

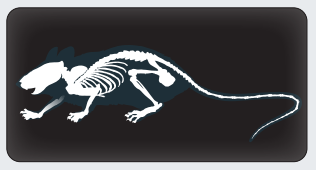

BLOOD-PRESSURE TEST

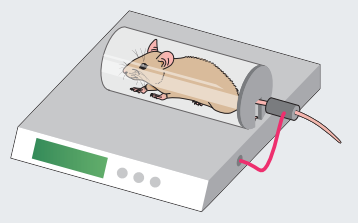

They should measure parameters such as blood pressure, metabolic rate, cardiac output and kidney filtration at animals' normal resting states and in response to common physiological challenges, such as running on an exercise wheel or completing a maze.

In the past decade, the US National Institutes of Health (NIH) has released a set of testing batteries for clinical research (see go.nature.com/invecq). These measures, including tests for balance, memory, strength and perception, can be used across many kinds of studies and are designed to make it easier to combine results from different trials.

Longitudinal studies in humans, such as the $\mathrm{NIH}$-supported Dynamics of Health, Aging, and Body Composition (Health ABC) Study, have collected body scans, health inventories, and physical performance data (such as walking speed) to establish clinically relevant measures of bone density, blood pressure and motor function. These kinds of assessments, adapted for rodents, should be used to determine which of hundreds of pharmacological compounds and nutritional interventions that are shown to slow the effects of ageing in model organisms should be advanced to trials in humans.

Investigators studying ageing in model organisms and humans must collaborate more. At the NIH, for example, basic and clinical research that relates to the biology of ageing are administered in separate programmes. To translate animal work into human treatments, the ageing-research community must rethink how all those involved communicate and interact, and are trained and funded.

The need for such crosstalk in ageing research has been stated frequently, with little effect. The development of standardized functional assessments that extend from model organisms to human populations may finally make it happen.

Douglas Seals is professor in the Department of Integrative Physiology at the University of Colorado Boulder in Boulder, Colorado, USA. Simon Melov is director of genomics at the Buck Institute for Research on Aging in Novato, California, USA. e-mail:seals@colorado.edu

1. Hung, W. W., Ross, J. S., Boockvar, K. S. \& Siu, A. L. BMC Geriatr. 11, 47 (2011).

2. Fontana, L., Partridge, L. \& Longo, V. D. Science 328, 321-326 (2010).

3. Ikeno, Y. et al. Pathobiol. Aging Age Relat. Dis. 3, 20833 (2013).

4. López-Otín, C., Blasco, M. A., Partridge, L., Serrano, M. \& Kroemer, G. Cell 153, 1194-1217 (2013).

5. Barzilai, N. et al. J. Clin. Endocrinol. Metab. 95, 4493-4500 (2010).

6. Mercken, E. M. et al. Aging Cell 12, 645-651 (2013).

7. Guevara-Aguirre, J. et al. Sci. Transl. Med. 3, 70ra13 (2011).

8. Kennedy, B. K. \& Pennypacker, J. K. Transl. Res. 163, 456-465 (2013).

9. Longo, V. D. \& Mattson, M. P. Cell Metab. 19, 181-192 (2014).

10.de Brito, L. B. et al. Eur. J. Prev. Cardiol. 21, 892-898 (2012).

11.Studenski, S. et al. J. Am. Med. Assoc. 305, 50-58 (2011).

12.Johnson, T. E. Exper. Gerontol. 48, 640-643 (2013) 13.Kenyon, C. J. Nature 464, 504-512 (2010).

14. Harrison, D. E. et al. Nature 460, 392-395 (2009)

15. Nadon N. L. et al. Age (Dordr.) 30, 187-199 (2008).

16. Dai, D.-F. et al. Circulation 119, 2789-2797 (2009)

17.Justice, J. N. et al. Age (Dordr.) 36, 583-595 (2014).

\section{CORRECTION}

The Comment 'Polio eradication hinges on child health in Pakistan' (Nature 511, 285-287; 2014) accidentally referred to Abbottabad as a village instead of a city. 